\title{
Ländliche Peripherie statt Zwischenstadt: Entwicklungsprobleme ländlicher Räume in Ostdeutschland. Das Land Brandenburg als Beispiel
}

\section{Christof Ellger, Berlin}

\section{Zur Entwicklung des ländlichen Raums in Deutschland in den 90er Jahren}

In den $80 \mathrm{er} \mathrm{Jahren,} \mathrm{und} \mathrm{verstärkt} \mathrm{in} \mathrm{den} \mathrm{90er} \mathrm{Jahren,} \mathrm{ist}$ wiederholt die These aufgekommen, dass der Prozess der räumlichen Entwicklung, d.h. die räumlichen Verschiebungen von Bevölkerung, Beschäftigung, Investitionen etc., am Ende des 20. Jahrhundert weniger zu Gunsten der Agglomerationsräume - wie in der langen Phase der Urbanisierung - sondern viel eher zu Gunsten der ländlichen Räume verläuft. Eine Umkehr der großräumlichen Entwicklungsdynamik wird konstatiert: weg von der großräumlichen Konzentration hin zu Dekonzentration, «Counter-Urbanisierung» oder «Disurbanisierung» (so BADE 1997 und früher schon vorsichtiger bei KANAROGLOU \& BRAUN 1992).

In derartigen räumlich-statistischen Analysen zur Entwicklung von Bevölkerung oder auch Beschäftigung wird herausgestellt, dass die ländlichen Räume - insbesondere auch die peripheren ländlichen Räume - die stärksten Wachstumsraten bei den Einwohnerzahlen aufweisen, aber auch bei Beschäftigtenzahlen, sowohl insgesamt als auch hinsichtlich neuer Wachstumsbranchen, etwa bei den haushalts- oder unternehmensorientierten Dienstleistungen (BADE 1997: 252ff.). Als Gründe für diese Dynamik werden insbesondere die deutlich verbesserte Verkehrsanbindung vieler ländlicher Räume in Deutschland wie auch die verstärkt hervortretenden Agglomerationsnachteile in den Verdichtungsräumen (zum Beispiel in Form von Flächenengpässen, Verkehrs- und Umweltproblemen) vermutet (BADE 1997: 254).

Zwei methodische Probleme zeigen sich bei dieser Art von Arbeiten zur Dynamik der ländlichen Räume: Zum einen wird beim Vergleich von Wachstumsraten häufig der Basiseffekt nicht bedacht, d.h. die Tatsache, dass Wachstumsraten bei gleicher absoluter Zunahme danach differieren, wie hoch die verschiedenen Ausgangswerte sind: Wenn die Bevölkerungszahl eines Verdichtungsraumes nur eine geringe prozentuale Zunahme zwischen zwei Zeitpunkten aufweist, kann diese Zunahme in absoluten Zahlen (Personen) ausgedrückt beträchtlich größer ausfallen als in einem verglichenen ländlichen Raum. Bei stark unterschiedlichen Ausgangsniveaus der Teilräume und insgesamt beträchtlicher Dynamik sollte deshalb eher mit Verschiebungen zwischen den Anteilen dieser Teilräume am Gesamtwert operiert werden. Zum anderen bestim- men natürlich vor allem Raumtypisierung und -abgrenzung, die einer Raumentwicklungsanalyse zugrunde gelegt werden, deren Ergebnis: Werden Verdichtungsräume und suburbane Räume eher eng definiert, dann nimmt es nicht wunder, wenn die Zuwächse im verbleibenden ländlichen Raum entsprechend hoch ausfallen. Werden urbane und suburbane Räume dagegen großzügig abgegrenzt, fallen die Zonen stärkster Bevölkerungszunahme in diese Bereiche und die Entwicklung im ländlichen Raum erweist sich als schwächer. Angesichts des mittlerweile fortgeschrittenen Entwicklungs- und Expansionsprozesses der städtischen Verdichtungsräume mit ihren suburbanen Umlandgebieten erscheint dabei für die westdeutschen Bundesländer letzteres Vorgehen eher angemessen. Eine genauere Unterscheidung zwischen «suburban» und «nicht mehr suburban», also »exurban» (im Sinne von Nelson \& Sanchez 1999), sowie im engeren Sinne «ländlich» müsste dabei sicher auch aktionsräumliche Verflechtungen mit dem Verdichtungsraumkern bzw. der Randzone der Verdichtungsräume sowie Lebensstilelemente der Bevölkerung berücksichtigen. Eine derartige Regionalisierung liegt jedoch nicht vor.

Hier hilft ansatzweise die Raumkategorisierung des Bundesamtes für Bauwesen und Raumordnung weiter, die für die Bundesrepublik Deutschland drei siedlungsstrukturelle Regionstypen («Agglomerationen», «verstädterte Räume» und «ländliche Räume») bzw. - hier verwendet - neun (kleinräumlicher ausgewiesene) Kreistypen unterscheidet (vgl. Abb. 1). Auf ihrer Grundlage können die wesentlichen Aspekte der räumlichen Bevölkerungsentwicklung in den 90er Jahren - die Daten beziehen sich auf den Zeitraum 1990-1997 - herausgearbeitet werden (vgl. Tab. 1):

In Westdeutschland, d.h. in den alten Bundesländern, wächst die Bevölkerung (durch Wanderungsgewinne aus der Ost-West-Wanderung und aus der Zuwanderung aus dem Ausland) um fast drei Millionen bzw. um fast $5 \%$. Dabei bleibt die Zunahme in den Kernstädten der großen Agglomerationsräume sowie der geringer verdichteten «verstädterten Räume» proportional weit hinter der durchschnittlichen Entwicklung zurück, so dass die Stadtkreise insgesamt ihren Anteil an der westdeutschen Bevölkerung beträchtlich reduzieren (die Kernstädte der großen Agglomerationen um über einen Prozentpunkt, die Kernstädte der verdichteten Räume um 0,2 Prozentpunkte). Überproportionale Zunahme weisen dagegen die Kreise in den äußeren Zonen der Agglomerationsräume auf und ganz besonders die verdichteten Kreise der verstädterten Räume, deren Anteil an der westdeutschen Gesamt- 


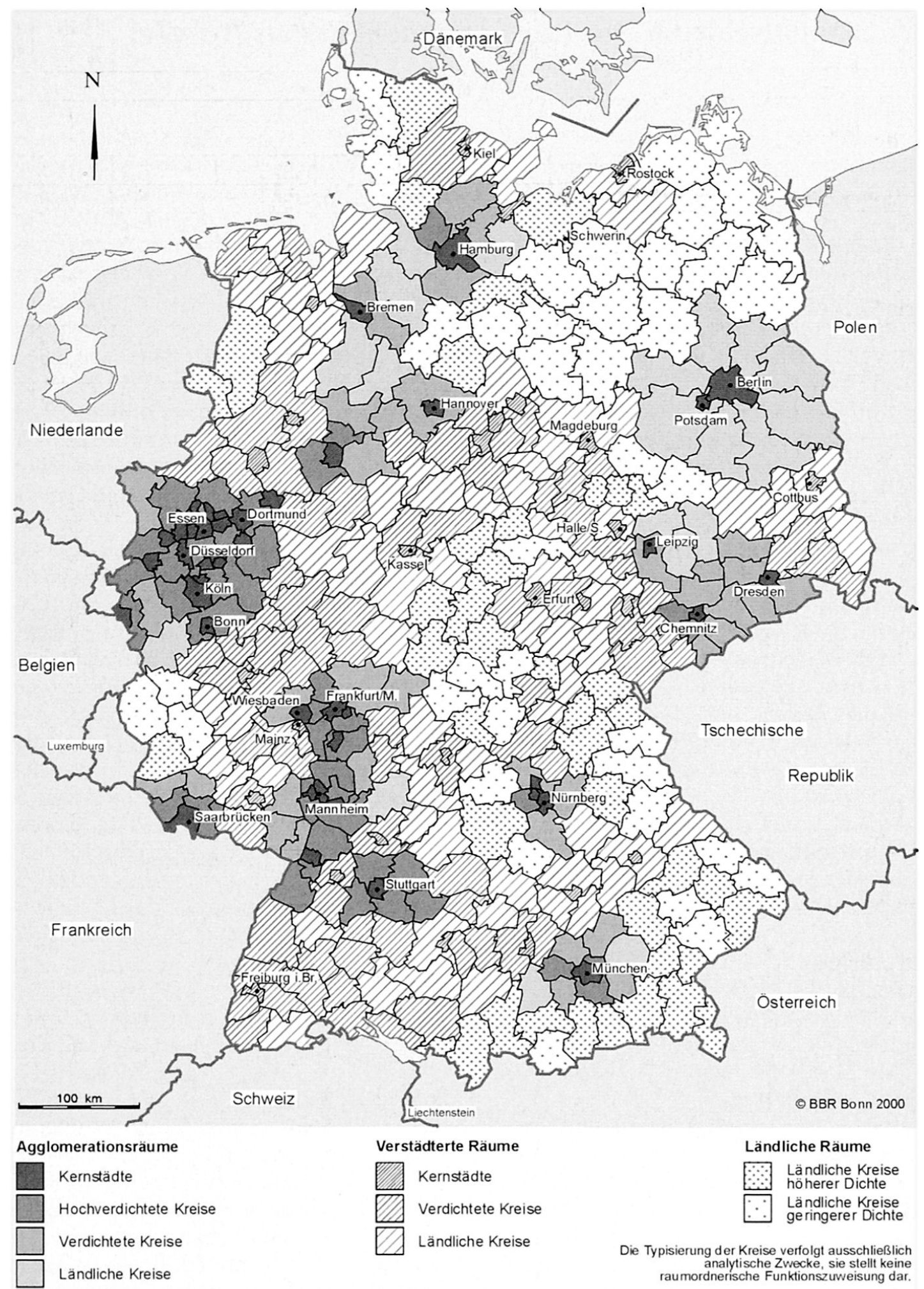

Abb. 1: Bundesrepublik Deutschland: Siedlungsstrukturelle Kreistypen 1997 Categories for spatial analysis in Germany, 1997

République fédérale d'Allemagne: Structures de peuplement par types d'arrondissements 1997

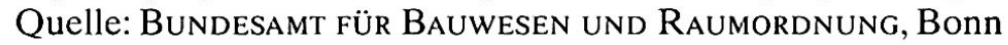




\begin{tabular}{|c|c|c|c|c|c|c|}
\hline & \begin{tabular}{|l|} 
Bevölkerung \\
1990 \\
Mio.
\end{tabular} & \begin{tabular}{|l} 
Bevölkerung \\
1997 \\
Mio.
\end{tabular} & \begin{tabular}{|l|} 
Differenz \\
$1990-1997$ \\
Mio.
\end{tabular} & \begin{tabular}{|l|} 
Entwicklung \\
$1990-1997$ \\
$\%$
\end{tabular} & \begin{tabular}{|l|} 
Anteil \\
1990 \\
$\%$
\end{tabular} & $\begin{array}{l}\text { Anteil } \\
1997 \\
\%\end{array}$ \\
\hline \multicolumn{7}{|l|}{ West } \\
\hline \multicolumn{7}{|l|}{ Agglomerationsräume: } \\
\hline Kernstädte & 14,8933 & 14,9270 & 0,0337 & 0,2 & 24,2 & 23,1 \\
\hline Hochverdichtete Kreise & 12,0637 & 12,7067 & 0,6430 & 5,3 & 19,6 & 19,7 \\
\hline Verdichtete Kreise & 5,1022 & 5,5000 & 0,3978 & 7,8 & 8,3 & 8,5 \\
\hline Ländliche Kreise & 1,3453 & 1,4714 & 0,1261 & 9,4 & 2,2 & 2,3 \\
\hline \multicolumn{7}{|l|}{ Verstädterte Räume: } \\
\hline Kernstädte & 3,3822 & 3,4284 & 0,0462 & 1,4 & 5,5 & 5,3 \\
\hline Verdichtete Kreise & 12,3619 & 13,2419 & 0,8800 & 7,1 & 20,1 & 20,5 \\
\hline ländliche Kreise & 5,6453 & 6,0767 & 0,4314 & 7,6 & 9,2 & 9,4 \\
\hline \multicolumn{7}{|l|}{ ländliche Räume: } \\
\hline höherer Dichte & 4,7389 & 5,0407 & 0,3018 & 6,5 & 7,7 & 7,8 \\
\hline geringerer Dichte & 2,0409 & 2,1553 & 0,1144 & 5,6 & 3,3 & 3,3 \\
\hline zusammen & 61,5737 & 64,5481 & 2,9744 & 4,8 & 100 & 100 \\
\hline ländliche Kreise insg. & 13,7704 & 14,7441 & 0,9737 & 7,1 & 22,4 & 22,8 \\
\hline \multicolumn{7}{|l|}{ Ost } \\
\hline \multicolumn{7}{|l|}{ Agglomerationsräume: } \\
\hline Kernstädte & 4,8722 & 4,7224 & $-0,1498$ & $-3,1$ & 26,8 & 27,0 \\
\hline hochverdichtete Kreise & 0,2591 & 0,2496 & $-0,0095$ & $-3,7$ & 1,4 & 1,4 \\
\hline verdichtete Kreise & 1,4085 & 1,3856 & $-0,0229$ & $-1,6$ & 7,7 & 7,9 \\
\hline ländliche Kreise & 1,7860 & 1,8364 & 0,0504 & 2,8 & 9,8 & 10,5 \\
\hline \multicolumn{7}{|l|}{ Verstädterte Räume: } \\
\hline Kernstädte & 1,5489 & 1,3695 & $-0,1794$ & $-11,6$ & 8,5 & 7,8 \\
\hline verdichtete Kreise & 2,3821 & 2,2635 & $-0,1186$ & $-5,0$ & 13,1 & 12,9 \\
\hline ländliche Kreise & 2,2080 & 2,1561 & $-0,0519$ & $-2,3$ & 12,1 & 12,3 \\
\hline \multicolumn{7}{|l|}{ ländliche Räume: } \\
\hline höherer Dichte & 1,4995 & 1,4206 & $-0,0789$ & $-5,3$ & 8,3 & 8,1 \\
\hline geringerer Dichte & 2,2151 & 2,1054 & $-0,1097$ & $-5,0$ & 12,2 & 12,0 \\
\hline zusammen & 18,1795 & 17,5091 & $-0,6704$ & $-3,7$ & 100 & 100 \\
\hline & & & & & & \\
\hline Ländliche Kreise insg. & 7,7087 & 7,5185 & $-0,1901$ & $-2,5$ & 22,4 & 22,8 \\
\hline
\end{tabular}

Tab. 1: Bevölkerungsentwicklung nach Raumkategorien (siedlungsstrukturelle Kreistypen) in Deutschland West und Ost, 1990-1997

Population change according to spatial categories in West and East Germany 1990-1997

Evolution de la population, selon les catégories spatiales (structures de peuplement par types d'arrondissements), en Allemagne de l'Ouest et de l'Est (1990-1997)

Quelle: Bundesamt Für Bauwesen und Raumordnung 
bevölkerung von $20,1 \%$ auf $20,5 \%$ steigt. Auch alle Typen von ländlichen Kreisen (zu Agglomerationen zugehörige ländliche Kreise, zu verstädterten Räumen zugehörige ländliche Kreise sowie ländliche Räume im engeren Sinn) wachsen überdurchschnittlich, so dass sich die Anteile dieser Raumkategorien an der Bevölkerung insgesamt vergrößern. Dabei wächst jedoch - aufgrund der vergleichsweise geringen absoluten Einwohnerzahl - der Bevölkerungsanteil all dieser als ländlich definierten Kreise lediglich von 22,4\% im Jahr 1990 auf 22,8\% im Jahr 1997.

Wesentliche Gewinner der Bevölkerungsverschiebungen in Westdeutschland in den 90er Jahren sind damit vor allem die verdichteten Kreise im weiteren Umland der Agglomerationskerne bzw. der Kerne der sonstigen verstädterten Räume. Diese Form der Raumentwicklung kann am besten als weitergeführte Suburbanisierung bezeichnet werden, sozusagen als «Sub-Suburbanisierung» bzw. Ex-Urbanisierung, als letztere vor allem dann, wenn die Bevölkerung dieser Räume zwar noch mit dem Agglomerationsraum in enger Interaktion steht, jedoch nicht vorrangig mit dem Kern, sondern nur noch mit den suburbanen «edge»Standorten des Arbeitens, der Versorgung oder der Freizeitaktivitäten. Die Verdichtungsräume dehnen sich unter Abnahme ihrer Dichte (vor allem im hochverdichteten Kern) nach außen hin aus und beziehen zunehmend vormals ländliche Bereiche in ihre Entwicklung mit ein. Die Welle der «Suburbanisierungsfront» dringt immer weiter in den ländlichen Raum vor. «Zwischenstädtische Räume» werden zunehmend aufgesiedelt und wachsen immer mehr zu (vgl. auch SiEVERTS 1999). Der Raumordnungsbericht 2000 erwähnt in diesem Zusammenhang die «Radiuserweiterung, zunehmende Siedlungsdispersion und eine zunehmende funktionale Anreicherung der Suburbanisierung» (Raumordnungsbericht 2000: 52). Der ländliche Raum als Raumkategorie im engeren Sinne «schwindet» zusehends bzw. zieht sich auf einzelne ländlichperiphere Flächen zurück.

Die räumlichen Verschiebungen bezüglich der Beschäftigung vollziehen sich noch dynamischer, insbesondere sind die anteilsbezogenen Verluste (vor allem durch rationalisierungsbedingten Arbeitsplatzabbau) in den Kernstädten noch höher als bei der Wohnbevölkerung. Die Verschiebungen kommen den anderen Raumkategorien in ähnlicher Weise wie bei den Einwohnerzahlen zugute, wobei die Umlandkreise um die Kernstädte in beiden Verdichtungsraumtypen noch am meisten Zuwachs abbekommen (BöLTKEN et al. 1999: 98ff.).

Ostdeutschland, d.h. die «neuen Bundesländer», befindet sich dagegen in den 90er Jahren des 20. Jahrhunderts noch in einer anderen Phase der räumlichen Entwicklung: in der einer großräumlichen Stärkung der Agglomerationsräume, in einer Verbindung aus Urbanisierung und Suburbanisierung. Dabei ist allerdings die Gesamtentwicklung von Abwanderung und
Bevölkerungsverlust gekennzeichnet (-3,7\% zwischen 1990 und 1997, das entspricht 670000 Einwohnern; Tab. 1). Stärkste Abnahmen weisen die Kernstädte der «verstädterten Räume» auf (Halle, Magdeburg, Rostock, Erfurt, Gera, Cottbus, Zwickau und Jena), also die Städte der zweiten Ebene des ostdeutschen Städtesystems, sowie die daran angrenzenden verdichteten Kreise, während die Kernstädte der (größeren) Agglomerationsräume Ostdeutschlands - Berlin, Leipzig, Dresden und Chemnitz - insgesamt weniger an Bevölkerung verlieren und ihren Anteil an der ostdeutschen Bevölkerung also vergrößern können, was das Etikett einer (relativen) «Urbanisierung» rechtfertigt. Absoluten Bevölkerungszuwachs weisen nur die ländlichen Kreise der großen Agglomerationsräume auf (bei diesen handelt es sich im Wesentlichen um die Umlandkreise von Berlin!). Ostdeutschlands ländliche Räume (im engeren Sinne) verlieren überdurchschnittlich stark an Bevölkerung (-5\% und mehr gegenüber -3,7\%; Tab. 1). Wenn die Gesamtentwicklung der ländlichen Räume in Ostdeutschland bei der Darstellung letztlich mit einem Anstieg von 22,4\% auf $22,8 \%$ leicht positiv ausfällt (zufällig sind die Werte identisch mit jenen für Westdeutschland), so nur, weil wegen der Ausweisung der «Tortenstück-Landkreise» um Berlin als «ländlich» die Berliner Umlandentwicklung, also der Suburbanisierungsprozess, hier als Verschiebung zugunsten des ländlichen Raums gerechnet ist.

Im Ergebnis ist die räumliche Dynamik in Deutschland in den 90er Jahren gekennzeichnet durch ein Vorherrschen von Sub- und Exurbanisierung in Westdeutschland und von Urbanisierung mit Suburbanisierung in Ostdeutschland. Der ländliche Raum in Westdeutschland wird unter den bestehenden Wachstumsbedingungen von der großflächigen Ausdehnung der Verdichtungsräume gleichsam «aufgezehrt», von dem immer größere Distanzen erfassenden Suburbanisierungsprozess überfahren und auf wenige Restflächen reduziert. In Ostdeutschland besteht weiterhin ein krasses Gefälle zwischen noch eher kompakten Verdichtungsräumen einerseits und großflächigen ländlichen Räumen andererseits (das dabei mit einem Gefälle zwischen Süd und Nord einhergeht). Weite Teile dieser ländlichen Räume sind von massiven Strukturproblemen gekennzeichnet. In Übereinstimmung damit zeigt sich gerade auch im Raum Berlin-Brandenburg eine deutliche Diskrepanz zwischen dem metropolitanen Innenraum einerseits und der strukturschwachen ländlichen Peripherie andererseits.

\section{Die Raumstruktur Brandenburgs: berlinnaher Wachstumsraum und strukturschwache Peripherie}

Für eine raumstrukturelle Darstellung Brandenburgs erscheint die BBR-Regionalisierung (Abb. 1) wenig 
brauchbar. Sie weist das ganze Bundesland - mit Ausnahme der Stadtkreise Potsdam und Cottbus - als ländlichen Raum aus (die acht Umlandkreise Berlins dabei als ländliche Kreise eines Agglomerationsraums Berlin). Dies ist nur insofern richtig, als im Raum Berlin-Brandenburg Deutschlands größte Stadt einerseits und das nach Mecklenburg-Vorpommern am spärlichsten besiedelte Bundesland Brandenburg andererseits aufeinandertreffen.

Abgesehen davon ist diese Darstellung der Raumstruktur Brandenburgs sicherlich zu undifferenziert. Insbesondere wird sie der besonderen Situation der berlinnahen Landesteile nicht gerecht. Die im suburbanen Umfeld Berlins gelegenen Bereiche unterliegen aktuell vielfach einem beträchtlichen Wachstumsdruck. Sie besitzen aufgrund der geringen Entfernung nach Berlin einen klaren Lagevorteil hinsichtlich der Erreichbarkeit von Arbeitsplätzen oder Versorgungs- bzw. Dienstleistungs-Einrichtungen aller Art. Von daher ist es angebracht, einen suburbanen Ring um Berlin in eine Raumstrukturdarstellung Brandenburgs mit aufzunehmen, zum Beispiel in der Abgrenzung des engeren Verflechtungsraums (Abb. 2), wie dies für die Zwecke der Gemeinsamen Landesplanung Berlin-Brandenburg unternommen worden ist (wenn auch sicherlich einige Teilbereiche am äußeren Rand des engeren Verflechtungsraumes durchaus noch sehr ländlich geprägt sind).

Vernachlässigt werden in einer derartigen räumlichen Gliederung aber auch die städtischen Anteile des brandenburgischen Außenraums: die Großstadt Cottbus (mit knapp 120000 Einwohnern), die Städte Brandenburg und Frankfurt an der Oder (mit Einwohnerzahlen um 80 000), Schwedt, Eberswalde und Eisenhüttenstadt (mit gut 40000 Einwohnern) sowie das disperse Städtenetz der Niederlausitz am Südrand des Bundeslandes (Abb. 2) mit seinen Bergbaufeldern und Industrieanlagen. Bei all diesen städtischen Zentren hat sich in den vergangenen zehn Jahren ein kleinräumiger Suburbanisierungsprozess abgespielt, gespeist von Bevölkerungsverschiebungen aus den Städten in die angrenzenden Landgemeinden.

Im Vorgriff auf den entstehenden «Landesentwicklungsplan für den Gesamtraum Berlin-Brandenburg (LEP GR)» ist im Agrarbericht 2000 des Landes Brandenburg eine Siedlungsstruktur-Darstellung aufgenommen, die der beschriebenen Differenzierung gerecht zu werden versucht (Bericht zur Lage der Land- und Ernährungswirtschaft des Landes Brandenburg 2000: 1) und den Planungsraum in drei Raumkategorien gliedert (Abb. 3):

1) verdichtete städtische Räume, von denen es vier gibt (Berlin mit Potsdam und weiteren Gemeinden, Cottbus, Frankfurt/Oder und Brandenburg an der Havel),

2) zentrennahe ländliche Räume (also suburbane Räume) um die verdichteten Räume sowie um wei- tere als Regionale Entwicklungszentren ausgewiesene Klein- und Mittelstädte des Landes,

3) alle übrigen Räume als ländliche Räume.

Trotz der Existenz von Verdichtungsansätzen in den genannten städtischen Konzentrationsräumen ist der «Außenraum» Brandenburgs - von der Landesplanung programmatisch (und euphemistisch) «äußerer Entwicklungsraum» genannt (Abb. 2) - im Wesentlichen ein dünnbesiedelter ländlicher Raum: Seine Bevölkerungsdichte beträgt 69 Einwohner $/ \mathrm{km}^{2}$. Wie wenig dies ist, zeigt sich im Vergleich mit dem Land Brandenburg insgesamt ( 88 Einwohner $/ \mathrm{km}^{2}$ ), der Bundesrepublik (230) oder auch der EU (116); am deutlichsten jedoch im Vergleich mit dem Wert für Berlin (3818 Einwohner $/ \mathrm{km}^{2}$ ). Im gesamten brandenburgischen Außenraum gibt es nur 32 Gemeinden mit mehr als 10000 Einwohnern (Abb. 2), dazu weitere 25 mit mehr als 5000 Einwohnern. Insofern erscheint es angemessen, raumstrukturellen Analysen des Landes Brandenburg und des Gemeinsamen Planungsraums Berlin-Brandenburg die Dichotomie zwischen dem Verdichtungsraum «innen» und der ländlichen Peripherie «außen» zugrunde zu legen. Dafür spricht auch, dass die stärker urbanen und industrialisierten Bereiche des äußeren Entwicklungsraumes (mit Cottbus, Frankfurt/Oder und Brandenburg/ Havel) von Strukturschwächen und Phänomenen des Abbaus und Wegbrechens in verschiedener Weise gekennzeichnet sind, die diese Räume in ihrer Problemlage den genuin ländlichen Räumen sehr ähnlich werden lassen.

\section{Probleme des brandenburgischen Peripherieraums}

Bei allen Schwierigkeiten, mit denen der engere Verflechtungsraum gegenwärtig konfrontiert ist (insbesondere die stagnierende Bevölkerungsentwicklung und die Arbeitsplatzverluste), weist dieser hochverdichtete Bereich im Zentrum Berlin-Brandenburgs gegenüber der brandenburgischen Peripherie immer noch einen beträchtlichen Entwicklungsvorsprung und ein unverhältnismäßig höheres Entwicklungspotenzial auf.

Die Situation des Außenraums Brandenburgs sieht dagegen ganz anders aus. Eine zusammenfassende Darstellung der Problemlage liefert der ehemals für Landwirtschaft und ländlichen Raum zuständige Landesminister:

«1. Die angespannte und sich weiter zuspitzende Lage der öffentlichen Haushalte reduziert die Spielräume zur Schaffung und Unterhaltung einer vielfältigen Infrastruktur erheblich.

2. Die geringe Wirtschaftsdynamik - besonders im produzierenden Bereich - bringt keine Perspektiven für eine durchgreifende Verbesserung des Angebotes von Arbeits- und Ausbildungsplätzen. 


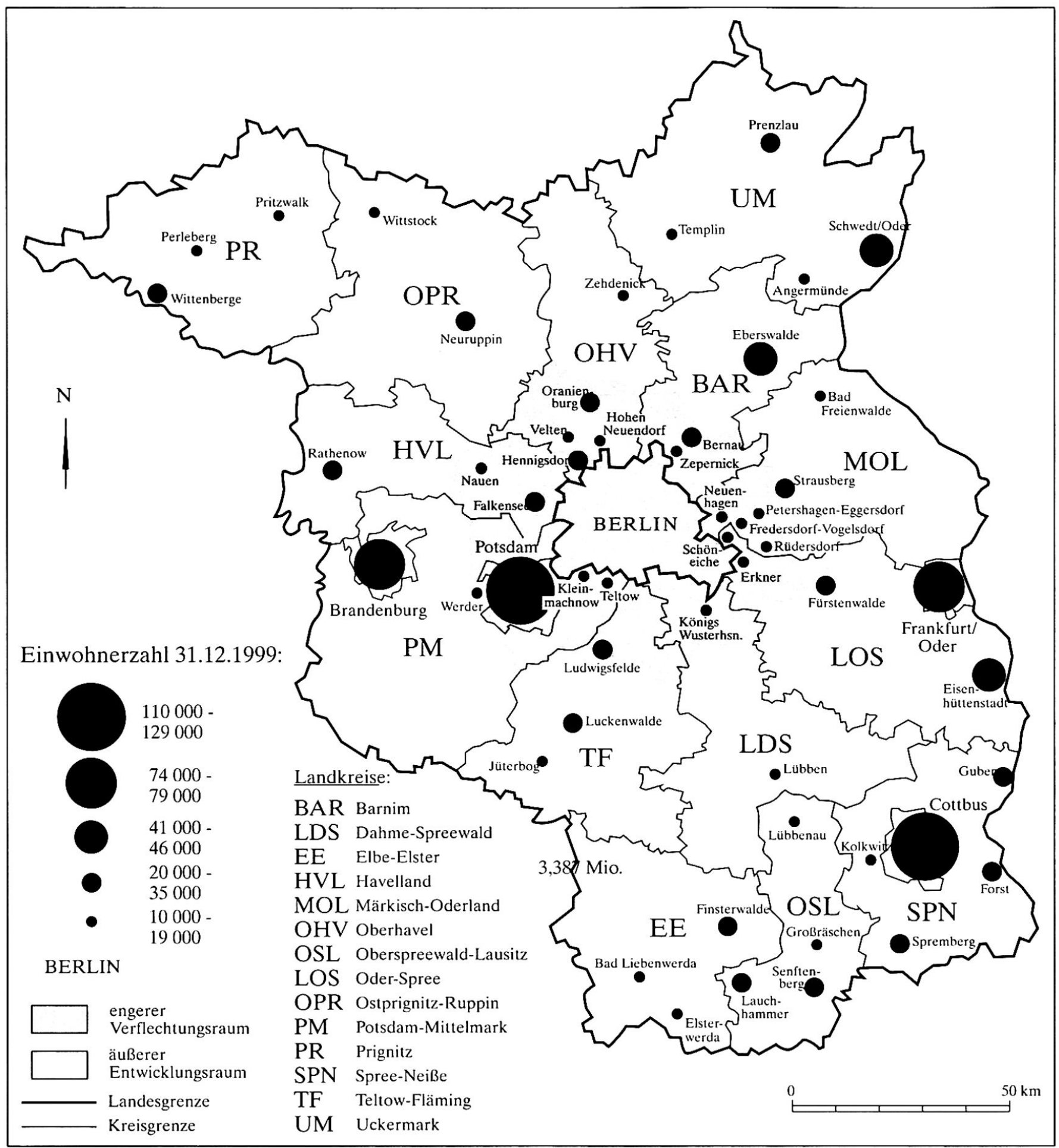

Abb. 2: Siedlungsstruktur Berlin-Brandenburg: Gemeinden mit mehr als 10000 Einwohnern, 1999 City system in the Berlin-Brandenburg area: communes with over 10000 inhabitants, 1999 Structure de peuplement à Berlin-Brandebourg: communes de plus de 10000 habitants (1999) Quelle: Landesbetrieb für Datenverarbeitung und Statistik (LDS) Brandenburg; Gemeinsame Landesplanungsabteilung Berlin-Brandenburg; Entwurf: C. Ellger, Kartographie: D. Engel 


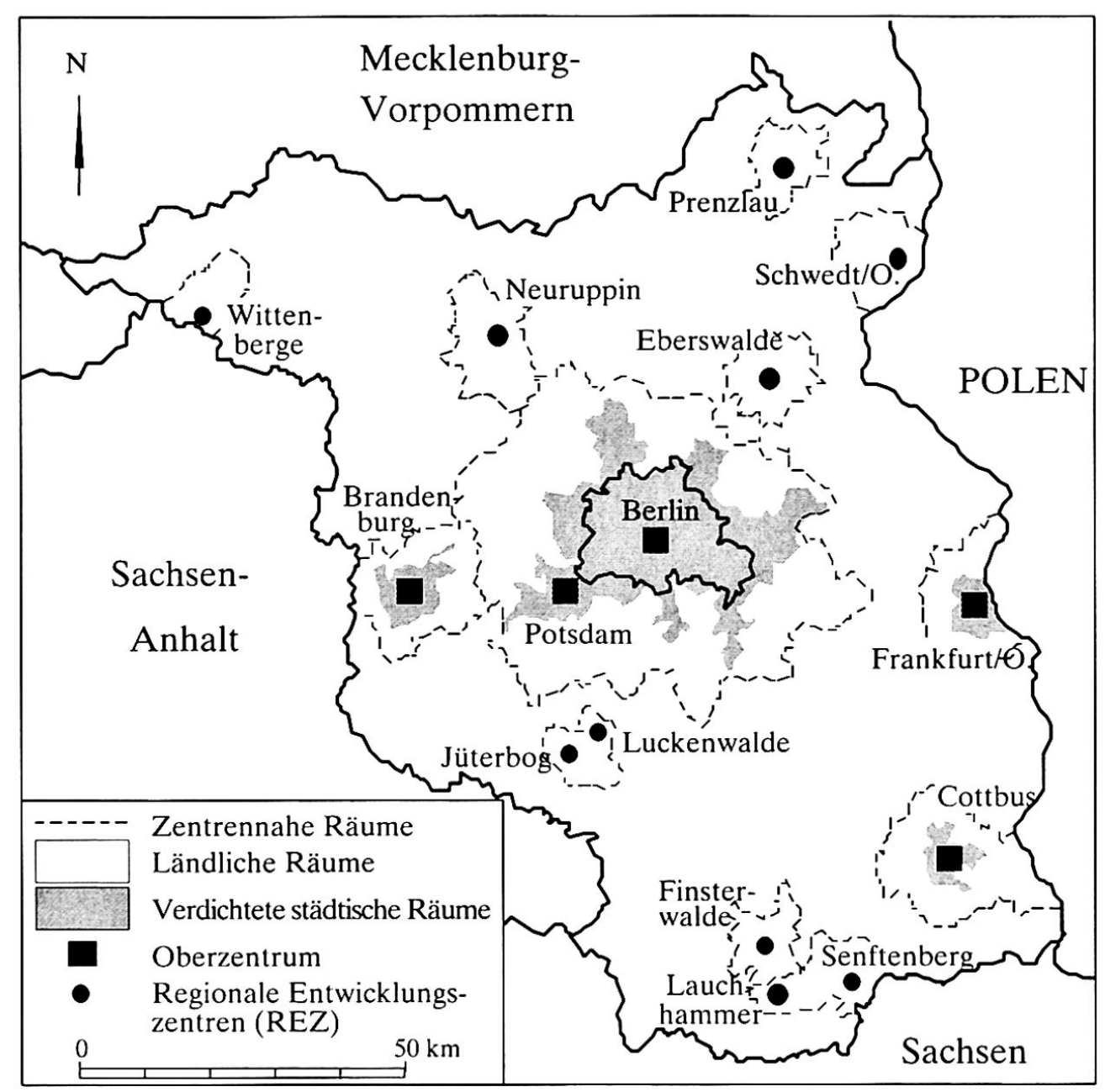

Abb. 3: Brandenburg: Raumkategorien der Landesplanung, 2000

Brandenburg: Spatial categories for planning purposes, 2000

Brandebourg: catégories spatiales d'aménagement du territoire (2000)

Quelle: Bericht zur Lage der Land- und Ernährungswirtschaft des Landes Brandenburg 2000, «Agrarbericht 2000»; Entwurf: C. ElLGER, Kartographie: D. ENGEL

3. Die demographische Entwicklung und Abwanderung, besonders in den ohnehin dünn besiedelten peripheren Räumen, verursachen einen Bevölkerungsrückgang und gefährden zunehmend die Tragfähigkeit einer vielfältigen Infrastruktur.

4. Hohe Arbeitslosigkeit und zu geringe berufliche Perspektiven für junge Menschen tragen zur Überalterung und Entleerung ländlicher Räume bei und begünstigen das Anwachsen von Konfliktpotenzial» (ZimmermanN 1997: 7).

Belegt werden kann der Gegensatz zwischen dem dynamischen Innenraum (engeren Verflechtungsraum) und dem zurückbleibenden bzw. in vieler Hinsicht sich zurückentwickelnden Außenraum («äußeren Entwicklungsraum») anhand der Darstellung von wesentlichen demographischen und wirtschaftlichen Indikatoren: In den 90er Jahren (10/1990-12/1999) hat die Ein- wohnerzahl im «Innenraum» Brandenburgs um 16,5\% zugenommen, im «Außenraum» dagegen um 7,2\% abgenommen, während die Bevölkerungszahl im Land insgesamt (nach Abnahme bis zum Tiefststand im Jahr 1994 und darauf folgender Zunahme) ungefähr konstant geblieben ist (Tab. 2). Der Anteil des berlinnahen Verflechtungsraumes an der Bevölkerungszahl des Landes hat sich dadurch von 30 auf $35 \%$ erhöht, der des äußeren Entwicklungsraumes entsprechend von 70 auf $65 \%$ reduziert. Mit ihren Anteilen am engeren Verflechtungsraum weisen alle acht an Berlin grenzenden Landkreise einen Bevölkerungszuwachs auf, während die sechs peripheren Landkreise im Norden und Süden des Landes - Prignitz, Ostprignitz-Ruppin, Uckermark, Elbe-Elster, Oberspreewald-Lausitz und Spree-Neiße - sämtlich einen Bevölkerungsrückgang zu verzeichnen haben (am geringsten im Spree-Neiße- 


\begin{tabular}{|c|c|c|c|c|c|}
\hline & $\begin{array}{l}\text { Bevölkerung } \\
3.10 .1990\end{array}$ & $\begin{array}{l}\text { Anteil am } \\
\text { Landeswert } \\
\text { in } \%\end{array}$ & $\begin{array}{l}\text { Bevölkerung } \\
31.12 .1998\end{array}$ & $\begin{array}{l}\text { Anteil am } \\
\text { Landeswert } \\
\text { in \% }\end{array}$ & $\begin{array}{l}\text { Veränderung } \\
1990-1998 \\
\text { in \% }\end{array}$ \\
\hline Land Brandenburg & 2602404 & 100 & 2601207 & 100 & $-0,5$ \\
\hline engerer Verflechtungsraum & 787038 & 30,2 & 916527 & 35,2 & $+16,5$ \\
\hline äußerer Entwicklungsraum & 1815366 & 69,8 & 1684680 & 64,8 & $-7,2$ \\
\hline Landkreis Prignitz & 109435 & 4,2 & 97076 & 3,7 & $-11,3$ \\
\hline Landkreis Ostprignitz-Ruppin & 118794 & 4,6 & 114273 & 4,4 & $-3,8$ \\
\hline Landkreis Uckermark & 170409 & 6,5 & 154086 & 5,9 & $-9,6$ \\
\hline Landkreis Elbe-Elster & 142679 & 5,5 & 132873 & 5,1 & $-6,9$ \\
\hline Landkreis Oberspreewald-Lausitz & 166351 & 6,4 & 148124 & 5,7 & $-11,0$ \\
\hline Landkreis Spree-Neiße & 157358 & 6,0 & 155247 & 6,0 & $-1,7$ \\
\hline Berlin-ferne Landkreise & 865026 & 33,2 & 801679 & 30,8 & $-7,3$ \\
\hline
\end{tabular}

Tab. 2: Bevölkerungsentwicklung in Brandenburg und ausgewählten Teilräumen Population change in Brandenburg and selected areas Evolution démographique dans le Land de Brandebourg et dans un choix de sous-régions Quelle: Statistisches Jahrbuch BRANDENBURG 2000

Kreis wegen der Suburbanisierung um Cottbus). Abseits der städtischen Verdichtungsbereiche und in größerer Entfernung zu Berlin liegen großflächige Entleerungsgebiete, die durch hohe Bevölkerungsverluste von mehr als $10 \%$ in den 90er Jahren gekennzeichnet sind. Zu diesen Räumen zählen die Prignitz, die nördliche Uckermark, weite Teile des Raumes entlang der polnischen Grenze und ländliche Räume in Südbrandenburg (ZimmermanN 1997: 19). Hintergrund ist in der Regel vor allem der hohe Sterbeüberschuss in den Landkreisen, weniger die Abwanderung. In der Prignitz, der Uckermark und im Landkreis Oberspreewald-Lausitz gewinnt letztere in den vergangenen Jahren mit hohen Abwanderungsraten jedoch die Oberhand.

Die Bevölkerungsprognose für Brandenburg für den Zeitraum 1997-2015 geht von einem weiteren Bevölkerungswachstum im brandenburgischen Teil des engeren Verflechtungsraums von $29,4 \%$ aus, dies entspricht einer absoluten Bevölkerungszunahme um gut 250000 auf 1,1 Mio. Einwohner, während der äußere Entwicklungsraum 200000 seiner rund 1,7 Mio. Einwohner (1997) - das sind 11,6\% - verliert (Bevölkerungsprognose für das Land Brandenburg 1999: 31). Damit wird sich im Außenraum auch die Auslastung der Infrastruktur weiter reduzieren, viele noch existierende Angebote werden aus wirtschaftlichen Gründen nicht mehr zu halten sein, was wiederum Erwerbsmöglichkeiten kostet, Einnahmen der öffentlichen Hand vermindert und die generelle Attraktivität dieser Räume weiter sinken lässt.

Das zentrale Problem des Außenraums Brandenburgs ist die höchst ungünstige Beschäftigungssituation. Die Arbeitslosenquote (berechnet auf der Basis der Einwohner zwischen 20 und 60 Jahren) hat sich im Zeitraum 1995-1997 von 12,3 auf 16,8\% erhöht. Dabei weisen die sechs berlinfernen Landkreise - trotz der Bevölkerungsabnahme - alle überdurchschnittliche Arbeitslosenquoten (berechnet nach den Vorgaben der Arbeitsverwaltung) auf (12/1998), zwischen 19,0\% (Ostprignitz-Ruppin) und 23,5\% (Oberspreewald-Lausitz), bei einem Landesdurchschnittswert von $17,7 \%$. 
Ohne die Förderung des zweiten Arbeitsmarkts lägen die Quoten noch bedeutend höher. Die gravierenden Beschäftigungsverluste in Landwirtschaft und Industrie in der Nachwendezeit konnten in keiner Weise durch neue Beschäftigungsmöglichkeiten - in welcher Branche auch immer - auch nur annähernd ausgeglichen werden. Die beachtlichen Dezentralisierungseffekte der Industrialisierung peripherer Räume durch die Industriestandortpolitik der DDR sind damit in vielen Fällen zunichte gemacht. Abgesehen von einzelnen inselhaften Wieder- und Neuentwicklungsstandorten (wie etwa in Schwarzheide, Schwarze Pumpe, Eisenhüttenstadt, Tschernitz oder zukünftig Brand) fehlt es heute im peripheren Brandenburg insbesondere an Arbeitsplätzen. Dieser Raum hat seine Bedeutung als Standortraum agrarischer oder industrieller Produktion weitgehend eingebüßt. Ein gravierender Stellenabbau in der Forstwirtschaft steht unmittelbar bevor. Und wirtschaftliche Führungsfunktionen, wie etwa Verwaltungen größerer Unternehmen oder unternehmensbezogene Dienstleistungen, existieren fast gar nicht, was mit der Art und Weise der Gestaltung des Transformationsprozesses in Ostdeutschland generell zusammenhängt, der Ostdeutschland ökonomisch in vieler Hinsicht zu einer abhängigen «Kolonie im eigenen Land» für Westdeutschland (bzw. Westeuropa) gemacht hat (Christ \& Neubauer 1993).

Die Steuereinnahmen der Kommunen haben sich im Land Brandenburg ebenfalls zwischen engerem Verflechtungsraum und äußerem Entwicklungsraum auseinander entwickelt: Im brandenburgischen Teil des engeren Verflechtungsraums ist das Ist-Steueraufkommen je Einwohner (aus der Grundsteuer, der Gewerbesteuer und dem Gemeindeanteil der Einkommensteuer) von 1992 bis 1997 von 313 DM auf $631 \mathrm{DM}$ gestiegen; im äußeren Entwicklungsraum dagegen hat der entsprechende Wert von 275 DM im Jahr 1992 auf 561 DM im Jahr 1995 zugenommen, danach jedoch wieder abgenommen, auf 448 DM im Jahr 1997 (Raumstrukturelle Entwicklungstrends im gemeinsamen Planungsraum Berlin-Brandenburg 1999: 13).

Dabei kann kein Zweifel daran bestehen, dass ohne die massive regionalpolitische Förderung der 90er Jahre und ohne den großflächig etablierten zweiten Arbeitsmarkt die prekäre wirtschaftliche Situation im ländlich-peripheren Raum Brandenburgs noch weit problematischer wäre.

Die Disparitäten zwischen dem entwicklungsstärkeren «Innenraum» und dem stagnierenden, zum Teil von Schrumpfung betroffenen "Außenraum» in Brandenburg kommen auch darin zum Ausdruck, dass die Bautätigkeit in den 90er Jahren sich ganz deutlich im engeren Verflechtungsraum konzentriert hat (Raumstrukturelle Entwicklungstrends 1999: Karte 3).

Wie die verbliebene Bevölkerung im ländlichen Raum Brandenburgs ihre Lebensbedingungen wahrnimmt und einschätzt - darüber ist wenig bekannt. In der
Fachdiskussion zu den ländlichen Räumen gilt neben dem Erwerbsproblem vor allem die Versorgung mit Dienstleistungen aller Art als ein Dauerproblem dieser Räume. Eine Untersuchung im zentrenfernen Raum des südwestlichen Landkreises Dahme-Spreewald ergab einen überraschend hohen Grad an Zufriedenheit mit den Lebensbedingungen im Allgemeinen sowie mit den Versorgungsmöglichkeiten im Besonderen (Ellger et al. 2000). 31 Prozent der Befragten sind sehr zufrieden mit den Lebensbedingungen an ihrem Wohnort und in dessen Umgebung, 62 Prozent sind «so ziemlich zufrieden», nur 7 Prozent sind «weniger zufrieden" und $0,4 \%$ sind "gar nicht zufrieden». Auch wenn man einen gewissen Effekt an «Euphemisierung», an (Sich-)Schönreden der Nachteile der eigenen Lebenswelt, berücksichtigt, erscheint die Zufriedenheit doch sehr stark ausgeprägt. Den Defiziten an Erwerbsmöglichkeiten und urbaner Abwechslung stehen eben auch Vorteile gegenüber, die erstere teilweise auszugleichen in der Lage sind: Genannt werden in erster Linie das Eigentum an Haus und Grund, die ländliche Ruhe, die Überschaubarkeit des dörflichen Lebens und die Nähe zur Natur. In ähnlicher Weise wurde die Erreichbarkeit von Dienstleistungen allgemein als wenig schwierig beschrieben und insgesamt konnten kaum Defizite in der Dienstleistungsausstattung ausgemacht werden. Diese Zufriedenheit hängt sicherlich sehr stark mit der hohen «Automobilisierung» zusammen: $93 \%$ der Befragten haben Zugang zu einem Pkw im Haushalt. Diese Zufriedenheit ist jedoch auch ein Resultat geringerer Ansprüche der Landbevölkerung, die zum einen sicherlich aller Ehren wert sind, andererseits jedoch auch - insbesondere wenn sie Kultur- und Bildungsaktivitäten betreffen durchaus problematisch zu sehen sind.

Bei einer derartigen Problemkonstellation, bei der die ländlichen Räume in verschiedener Hinsicht zurückbleiben bzw. sich sogar zurückentwickeln, besteht die Gefahr, dass sich im ländlichen Raum die Negativfaktoren gegenseitig verstärken und die Dynamik einer Abwärtsspirale entsteht, bei der der Rückgang bei einer Größe - sei es Bevölkerung, Beschäftigung, Investitionen der Wirtschaft, regionale Kaufkraft, Infrastrukturauslastung, öffentliche Einnahmen oder öffentliche Investitionen in Infrastruktur - weitere Rückgänge bei den anderen Größen nach sich zieht, mit entsprechenden Folgewirkungen in einer nächsten Runde. Damit drohen in weiten Teilen der brandenburgischen Peripherie kumulative Entleerungs- und Erosionserscheinungen in der sozioökonomischen Ausstattung.

\section{Entwicklung ja - aber welche?}

Es ist illusionär anzunehmen, im äußeren Entwicklungsraum Brandenburgs sei - bei ausreichender 
Förderung - eine Entwicklung denkbar, die die Disparitäten, die gegenüber dem engeren Verflechtungsraum (d.h. Berlin und dem angrenzenden Umland) bestehen, aufheben könnte. Zur Begründung dieser These lassen sich verschiedene Gesichtspunkte anführen:

Die wesentlichen Wachstumsbranchen der Gegenwart und nahen Zukunft sind hinsichtlich ihrer großräumlichen Standortorientierung im Wesentlichen auf Verdichtungsräume ausgerichtet. Es handelt sich dabei häufig um Wirtschaftszweige im Überschneidungsbereich von Produzierendem Gewerbe und Dienstleistungen, wie zum Beispiel die MultimediaBranche. Unternehmen dieser Bereiche sind auf intensive Vernetzung mit Kunden einerseits und Partnern aus der gleichen oder benachbarten Branchen andererseits angewiesen. Sie fordern ein «informationsreiches» Umfeld - aus baulichen Elementen und Menschen als tatsächlichen und potenziellen Interaktionspartnern -, das sie den aktuellen Entwicklungen in Wirtschaft und Gesellschaft, bei Produktionsformen und Konsumweisen, nahebringt und neue Ideen stimuliert, die die Entwicklung weiter vorantreiben.

Größere Unternehmen mit entsprechend größerem Platzbedarf weichen in den suburbanen Raum aus, der im Falle von Brandenburg in geradezu übertrieben ausreichendem Maße Flächen zur Verfügung stellen kann.

Die traditionellen Branchen, die zur Zeit die Wirtschaftsstruktur des ländlichen Brandenburgs kennzeichnen, wie zum Beispiel die Bauwirtschaft, kämpfen dagegen eher ums Überleben, als dass sie neue Impulse bringen könnten. Angesichts der Neuorientierung der EU-Agrarpolitik (Agenda 2000) ist auch die spezifische Ausprägung der Landwirtschaft in Brandenburg, die mit ihrem «Brandenburger Weg» in hohem Maße an den früheren Betriebsgrößen und -organisationsformen angeknüpft hat, tendenziell von einer Verschlechterung ihrer Wettbewerbsposition betroffen.

Die vielfältigen Konversionsaufgaben im Außenraum Brandenburgs erschweren dabei noch die Lage: Für die vielen ehemals militärisch genutzten Flächen müssen Nachnutzungen gefunden werden, die zum Teil auch die Sanierung der Flächen mit zu finanzieren in der Lage sind. Und auch der Umbau der Niederlausitz von einer dominant bergbaulich und energiewirtschaftlich geprägten Region zu einem Wirtschaftsraum mit einer anderen Struktur, die sich dabei erst in Ansätzen zeigt, ist unter «Konversion» zu fassen, in diesem Fall nicht militärischer sondern bergbaulicher Flächen.

Angesichts der im Vergleich zu (anderen) Metropolenräumen Europas eher schwachen Entwicklung Berlins (gemessen etwa an Bevölkerungszuwachs, Beschäftigungsentwicklung, Wachstum des Sozialprodukts o.ä.) ist mit einer überschäumenden Agglomerationsraum-Entwicklung, im Sinne der oben beschriebenen «Sub-Suburbanisierung» auch nicht zu rechnen. Die gegenwärtigen Suburbanisierungserscheinungen beschränken sich auf den engeren Verflechtungsraum (NowOSSADECK 1997: 30), und selbst dabei schwerpunktmäßig auf die inneren Bereiche. Für einen Prozess der Exurbanisierung, der in Teilräumen Westdeutschlands, wie dargestellt, nachgewiesen werden kann, fehlt im Raum Berlin-Brandenburg schlichtweg der Entwicklungsdruck. Die Dynamik im Kernraum Berlin ist viel zu schwach, als dass sie weit in die Peripherie ausstrahlen könnte (KRÄTKE \& BORST 2000: 285ff.): Es scheint nicht wahrscheinlich, dass sich aus dem - im Vergleich mit anderen (west-)deutschen Agglomerationsräumen zurückhängenden - Bestand der berlin-brandenburgischen Wirtschaft wie auch durch Ansiedlungen von außerhalb in nennenswertem Ausmaß neue Betriebsstätten für die Peripherie Brandenburgs rekrutieren ließen.

Das heißt nicht, dass es nicht auch Ausnahmen von den beschriebenen Tendenzen geben kann, die natürlich angesichts der Entwicklungsschwäche im Brandenburger Außenraum auch unbedingt zu nutzen sind. Es kann Nischen geben, wo ganz bestimmte Branchen Flächen und Standorte nachfragen, die nur der ländliche Raum Brandenburgs bieten kann. Solch eine Situation zeigt sich bei der Ansiedlung der «Cargolifter»-Luftschiffwerft bei Brand im Landkreis Dahme-Spreewald. Hier benötigt (endlich einmal) ein neues Unternehmen mit einem einzigartigen und in der Tat höchst innovativen Produkt - große Produktions- und Freiflächen, wie sie sich nur im ländlichen Raum finden lassen (in diesem Fall sogar als Konversionsflächen), wobei die Verkehrsanbindung über Autobahn und Schiene gewährleistet ist und die Entfernung zur Metropole Berlin für die Betreiber überwindbar bleibt.

Ebenso wichtig ist es, die bestehenden Produktionseinheiten und -strukturen so weit es geht zu erhalten und $-z$. B. durch stärkere Vernetzung - weiterzuentwickeln, was die Industriepolitik des Landes sich auch schwerpunktmäßig vorgenommen hat.

Insgesamt sind jedoch die Chancen einer industrialistischen, urban orientierten Entwicklungsstrategie für die ländlichen Räume Brandenburgs, die in der Tradition der Wirtschaftsförderung der Nachkriegszeit vor allem auf die Ansiedlung von Großeinheiten im Produzierenden Gewerbe setzt, eher gering einzuschätzen. Ähnliches gilt für industriell betriebene Landwirtschaft - und ganz offensichtlich auch für industrialisierte Formen von Tourismus in Form von Center Parks o.ä., von denen im Land bisher noch kein einziger realisiert worden ist. Mit ihrer Prägung durch den Braunkohlebergbau und die daran anknüpfenden Folgeindustrien (Verstromung und Grundstoffchemie) ist dabei die Niederlausitz als ein Sonderfall bezüglich der Regionalentwicklung zu sehen. Dieser besonderen Situation wird ja auch durch besondere Leitbilder und Entwicklungsstrategien - zum Beispiel dem der «IBA Fürst-Pückler-Park»-Rechnung getragen. 
Ein Entwicklungsweg für die ländliche Peripherie Brandenburgs ist nicht anders denkbar als dass er in erster Linie die geringe anthropogene Nutzungsintensität des Raumes inwertsetzt, d.h. die Naturlandschaft und die besonderen Naturschätze, und diese einsetzt für Zwecke der Erholung und des Naturerlebens. Im Mittelpunkt des Interesses stehen dabei natürlich die Wald-Seen-Gebiete des Landes. Dabei wird Brandenburg sicherlich nie ein Land für die großen Urlaubsreisen sein. Machbar erscheint dagegen ein Kurz-, Tages-, Sport-, Seminar- und Erholungs-Tourismus, wie er bereits Tradition besitzt, jedoch durchaus noch ausbaufähig erscheint. Quellraum für die Besucher bildet natürlich vorwiegend die Agglomeration Berlin. Dabei ist es wünschenswert, die Besucher stärker in die Verantwortung für die ländlichen Siedlungsräume einzubinden, was am besten durch (Zweit-)Hausbesitz im baulichen Bestand der betreffenden Erholungsräume zu bewerkstelligen ist, der Initiative und bauliche Erneuerung zurück in die Dörfer bringen kann. Zu denken wäre darüber hinaus auch an die Initiierung von (eher informellen) Partnerschaften zwischen Berliner Bezirken, Stadtteilen und Vereinen einerseits und Teilräumen in der Brandenburger Peripherie andererseits, die «menschliche Brücken» schaffen, über die Gedankenaustausch, aber auch Unterkunftsmöglichkeiten, Freizeitaktivitäten und sonstige gegenseitige Hilfe vermittelt werden könnten. Derartige Initiativen wären schon zu Anfang der 90er Jahre, am Beginn der «neuen Koexistenz» der beiden Länder Berlin und Brandenburg erforderlich gewesen.

Unabdingbar sind Erhaltung, Pflege und Entwicklung der Natur, damit diese die genannten Aufgaben auch auf sich nehmen kann. Dazu gehören auch Zonierungskonzepte und Maßnahmen der Besucherlenkung. Hierin liegt der besondere Stellenwert der Großschutzgebiete, die für großflächig definierte Landesteile sektorübergreifend planen und entwickeln können.

Was auch der Verfassungsauftrag und das Raumordnungsgesetz fordern, nämlich die Gewährleistung gleichwertiger Lebensbedingungen, muss die Politik für die ländlichen Räume leisten. Dies wird in Zukunft heißen: eine Grundsicherung im Reproduktionsbereich und die Unterstützung von Initiativen zur Selbsthilfe bei der Linderung der Arbeitslosigkeit. Bei vielen Gesprächen im ländlichen Raum deutet sich auch an, dass verstärkte Anstrengungen im Bereich von Bildung und vor allem auch Fort- und Weiterbildung notwendig erscheinen, die über unterschiedlichste Träger - von Volkshochschulen über Vereine, Kirchen, bis hin zu Betrieben oder die Feuerwehr als tragende Institutionen des Dorflebens - und in noch zu entwickelnden Formen angeboten werden müssen, damit sie eine Chance habe, ihre Bestimmungspersonen zu erreichen.

\section{Literatur}

BADE, F.-J. (1997): Zu den wirtschaftlichen Chancen und Risiken der ländlichen Räume. - In: Raumforschung und Raumordnung 55:247-259.

BöLtKEN, F. et al. (Bearb.) (1999): Aktuelle Daten zur Entwicklung der Städte, Kreise und Gemeinden. Ausgabe 1999. - = BBR-Berichte 3, Bonn.

Christ, P. \& R. Neubauer (1993): Kolonie im eigenen Land: die Treuhand, Bonn und die Wirtschaftskatastrophe der fünf neuen Bundesländer. - Reinbek: Rowohlt.

Ellger, C. und Mitglieder der studentischen Arbeitsgruppe «Projekt Luckau» (2000): Dienstleistungen im ländlichen Raum - Versorgung aus der Sicht der Nutzer, räumliche Verflechtungsmuster und zentrale Orte, untersucht im südlichen Landkreis DahmeSpreewald. Projektbericht. $-=$ METAR 38, Berlin.

Kanaroglou, P.S. \& G.O. Braun: (1992): The pattern of counterurbanization in the Federal Republic of Germany, 1977-85. - In: Environment and Planning A 24: 481-496.

KRÄTKE, S. \& R. Borst (2000): Berlin. Metropole zwischen Boom und Krise. - Opladen: Leske + Budrich.

Landesamt für Datenverarbeitung und Statistik Brandenburg und Landesumweltamt (1999): Bevölkerungsprognose für das Land Brandenburg 1998-2015. - Landesamt für Datenverarbeitung und Statistik Brandenburg, Potsdam.

LANDESUmWeltamt (1999): Raumstrukturelle Entwicklungstrends im gemeinsamen Planungsraum Berlin-Brandenburg. $-=$ Fachbeiträge des Landesumweltamtes 44, Potsdam.

Ministerium FÜR LANDWIRTSCHAFt, UmWeltschutZ und RaUmordnung DEs LaNdes BRandenburg (2000): Bericht zur Lage der Land- und Ernährungswirtschaft des Landes Brandenburg 2000, «Agrarbericht 2000». - Ministerium für Landwirtschaft, Umweltschutz und Raumordnung des Landes Brandenburg, Potsdam.

Nelson, A.C. \& T.W. Sanchez (1999): Debunking the exurban myth: a comparison of suburban households. - In: Housing Policy Dabate 10: 689-709.

NowOSSADECK, E. (1997): Suburbanisierungsprozesse in der Region Berlin-Brandenburg. - In: MARETZKE, S. (Hrsg.): Regionalbarometer neue Länder. Dritter zusammenfassender Bericht. - = Materialien zur Raumentwicklung 83, Bonn: 30-33.

RAUMORDNUNGSBERICHT 2000 (2000). - = BBR-Berichte 7, Bonn, Bundesamt für Bauwesen und Raumordnung.

SieverTs, T. (1999): Zwischenstadt: zwischen Ort und Welt, Raum und Zeit, Stadt und Land. - = BauweltFundamente 118, Braunschweig: Vieweg.

Zimmermann, E. (1997): Große Anfrage Nr. 23. Gegenwart und Zukunft des ländlichen Raumes. Redebeitrag von Agrarminister Edwin Zimmermann. - Ministerium für Ernährung, Landwirtschaft und Forsten des Landes Brandenburg, Potsdam. 


\section{Zusammenfassung: Ländliche Peripherie statt Zwischenstadt: Entwicklungsprobleme ländlicher Räume in Ostdeutschland. Das Land Brandenburg als Beispiel}

Der Wandel der Raumstruktur in Deutschland vollzieht sich im West- und Ostteil der Bundesrepublik in höchst unterschiedlicher Weise: Auf der Basis der Bevölkerungsentwicklung in den siedlungsstrukturellen Kreistypen kann gezeigt werden, dass in Westdeutschland unter Wachstumsdruck ein Prozess fortschreitender Suburbanisierung und Exurbanisierung abläuft, während in Ostdeutschland im Zuge von Urbanisierung und Suburbanisierung die Entwicklung stärker auf die Agglomerationsräume konzentriert bleibt. Damit finden sich ländliche Räume in den ostdeutschen Bundesländern in einem Prozess des Schrumpfens wieder, der ihnen verstärkt die klassischen Züge ländlich-peripherer Strukturschwäche aufzwingt. Dies kann für die ländlichen Räume Brandenburgs nachgewiesen werden. Entwicklungsstrategien hier können nicht anders als auf endogene Potenziale setzen und sollten darüber hinaus Initiativen zu Partnerschaften zwischen dem hoch urbanisierten Zentrum Berlin und der ländlichen Peripherie in Gang setzen.

\section{Summary: Rural Periphery Instead of Interurban Space: Problems of development of rural areas in Eastern Germany. The federal state of Brandenburg as an example}

Spatial change in Germany differs markedly between East and West:The data concerning population change in the different categories for spatial analysis developed by the Bundesamt für Bauwesen und Raumordnung shows that Western Germany is experiencing a process of advanced suburbanisation and exurbanisation. On the other hand, in East Germany, due to continued urbanisation and suburbanisation, urban agglomerations are still the dominant growth centres. Thus, rural areas in Eastern Germany are subject to a process of decline which shows the classical features of peripheral weakness. The state of Brandenburg surrounding Berlin is such an example. Development strategies cannot but rely on the endogenous potential in these areas and should, in addition, foster partnership agreements between the inhabitants of the urbanized centre of Berlin on the one hand and of its rural periphery on the other hand.
Résumé: Une périphérie rurale et non un espace interurbain: problèmes de développement des espaces ruraux en Allemagne de l'Est, à l'exemple du Land de Brandebourg

La transformation des structures spatiales s'effectue de manière très différente dans les parties occidentale et orientale de la République fédérale: l'évolution démographique des arrondissements (Kreise) catégorisés selon leur structure de peuplement permet de démontrer qu'en Allemagne occidentale la croissance engendre un processus de suburbanisation et d'exurbanisation, alors qu'en Allemagne orientale l'urbanisation et la suburbanisation restent plutôt confinées aux périmètres d'agglomérations.

Par conséquent les espaces ruraux des Länder d'Allemagne de l'Est accusent un déclin croissant qui leur impose les déficiences classiques propres aux territoires ruraux périphériques, ce qui peut être démontré par l'exemple du Brandebourg.

Les stratégies de développement retenues n'ont d'autre choix que de miser sur des potentiels endogènes et se doivent en plus de créer des partenariats entre le centre fortement urbanisé de Berlin et sa périphérie rurale.

Dr. Christof Ellger, Institut für Geographische Wissenschaften der Freien Universität Berlin, Malteserstrasse 74-100, D-12249 Berlin.

e-mail: cellger@geog.fu-berlin.de 